\title{
Obituary for Dr. Peter Werle
}

\author{
F. D'Amato ${ }^{1}$, A. Fried ${ }^{2}$, and E. Kerstel ${ }^{3}$
}

${ }^{1} \mathrm{CNR}$ - Istituto Nazionale di Ottica, Firenze, Italy

${ }^{2}$ Institute of Arctic and Alpine Research, University of Colorado, Boulder, USA

${ }^{3}$ Laboratory of Interdisciplinary Physics, Université J. Fourier, Grenoble, France

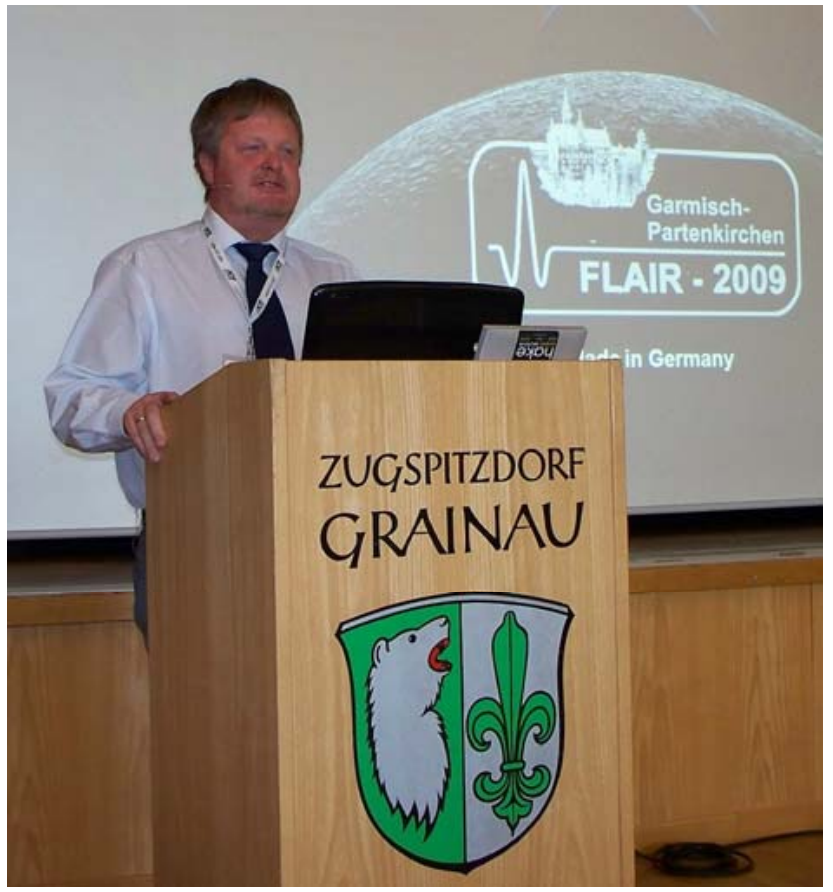

It is with great sadness and shock that we learned of having lost, on Friday 6 September 2013, a great friend, father, husband, scientist, mentor, symposium organizer, and a revolutionary intellectual thinker, who had the remarkable ability of addressing and describing fundamental problems in applied laser physics in new and enlightening ways. Although it is impossible to capture the full extent of Peter Werle's impacts in such a short space adequately, this tribute to Peter attempts to highlight his vast scientific contributions in numerous areas.

Peter Werle was born in Kaiserslautern in 1958. He studied physics at Johannes Gutenberg Universität in Mainz and received his Dr. rer. nat. degree in physical chemistry from Ludwig Maximilians Universität in Munich, with summa cum laude for his thesis work on quantum-limited laser spectroscopy. True to his self-reliant and resourceful nature, he paid his way through his studies by working on a freelance basis for IBM; at the time, this certainly was not very common in Germany. He headed different research groups at the Institute for Meteorology and Climate Research (IMK-IFU) in Garmisch-Partenkirchen, focusing on laser spectroscopy and integrated systems for environmental research. Peter brought this diverse background, combining outstanding experimental and mathematical skills, to atmospheric studies in the 1980s, employing liquid-nitrogen-cooled lead-salt diode lasers. At the time, such devices were fraught with many problems, and Peter methodically and systematically began to address each issue. He was particularly adept at introducing advanced algorithms and signal processing strategies and new data quality assurance concepts to diode laser measurements. In 1993 Peter and colleagues published a seminal paper in Applied Physics B in which they introduced the concept of Allan variance applied to laser diode measurements of gas concentration time series. The principle of using Von Neumann's two-sample variance to describe the statistics of time series that exhibit power law spectra that are more dispersive than white noise (e.g., show a varying mean, or drift) was first made popular by D. W. Allan in 1966 for characterizing frequency standards. The importance of introducing this new tool to laser physicists and atmospheric scientists cannot be overstated; it provided a wealth of new information in a mathematically formal and rigorous framework that would not otherwise be easily accessible. Today virtually every group in the world carrying out ultra-sensitive laser spectroscopic measurements employs the concepts introduced by Peter and colleagues. It is indeed rare to find a high-quality paper discussing laser spectroscopic measurements without an Allan plot. Perhaps such plots should in the future be referred to as "Allan-Werle" plots.

Peter Werle continued to push the frontiers of laser spectroscopic measurements and approaches for atmospheric studies. It was apparent that although tunable diode-laser 
measurements provided very high sensitivity, applications for real-world atmospheric research required significant additional improvements in system performance and stability. A technique frequently referred to as FM spectroscopy became popular in the late 1980s and early 1990s as a potential means to achieve this goal. A number of researchers reported quantum-limited detection limits 2-3 orders of magnitude better than traditional low-frequency $2 \mathrm{f}$ modulation approaches utilizing single-pass absorption cells in stable laboratory settings. Peter Werle questioned whether such improvements were indeed feasible with real-world detection systems, which required multiple-pass absorption cells with more extensive transfer optics to match the fast emerging diode laser beams to such multipass cells. Peter's instinct in not "following the herd" and simply blindly accepting the potential merits of FM spectroscopy is one of many examples of how Peter Werle advanced the field of laser spectroscopy as applied to atmospheric measurements. In a number of seminal papers, Peter and colleagues thereafter showed that pressure fluctuations in practical multiple-pass cells, arising from turbulent gas flow, reduced the expected enhanced performance of FM techniques. Furthermore, Peter and colleagues also determined that such FM spectrometers required extreme mechanical stability of the numerous optical components and that this further reduced the actual enhancements achievable in real-world FM laser spectrometers to about one order of magnitude or less over more traditional low-frequency $2 \mathrm{f}$ modulation techniques, at the expense of a great deal of additional complexity. To accomplish this, Peter Werle published an excellent paper whereby he considered the FM spectrometer as an interferometer and provided convincing evidence that enhancements in reducing excess laser noise in FM spectroscopy are achieved at the expense of reduced system stability. This again demonstrated Peter's remarkable ability to look at fundamental problems in laser physics in a completely different light. As a result of these advances, most laser spectrometers today now employ higher frequency $2 \mathrm{f}$ modulation approaches, thus striking the best compromise between FM and traditional $2 \mathrm{f}$ methods.

Peter continued to investigate and publish numerous papers throughout the 1990s on real time signal processing concepts for trace gas analysis. At the turn of the century, Peter's interests and career focus began to shift to other areas of research: solar UV radiation at the Network for Detection of Atmospheric Composition Change at one of the primary sites on top of Zugspitze in Germany; field studies on atmosphere-ecosystem exchange of trace gases over complex terrains; laser sensing at the land-atmosphere interface with a focus on peatlands; integrated systems for multi-scale studies of trace gas fluxes, turbulence and advection; as well as radiation variability inside canopies. His tireless efforts to assess critically the true merits of performance claims in the literature serve to illustrate Peter's intellectual honesty, without which we could not have advanced the state of the art in laser spectroscopy measurements. As one example, Peter was concerned that the commercial success of laser spectroscopic trace gas and isotope ratio analyzers by the atmospheric monitoring communities would lead to a proliferation of such devices, with a sizeable fraction of them ending up with end users having no idea of how to assure the quality of their data. In typical Peter Werle fashion, he addressed this concern by organizing special conference sessions and short courses devoted to this subject.

In addition to his extensive research resume, Peter Werle provided extensive service to the science community by serving as a guest editor of Applied Physics B, as an associate editor of Atmospheric Measurement Techniques, and most notably, starting in 2007, as the founding member of the Field Laser Applications in Industry and Research (FLAIR) conferences. This highly visible and successful series of conferences, which continue today, brings together industrial laser developers, users, and manufacturers with academic and government-funded researchers. This is yet another example of Peter Werle as a true visionary. Peter also loved to help make these FLAIR conferences as memorable as possible, not only for the scientific exchange during the meetings but also for the invaluable exchange during the numerous social events that he and Francesco D'Amato spent endless hours organizing. In recognition of his achievements, Peter Werle was appointed as a research associate at the National Institute of Applied Optics in Florence, Italy.

Aside from his copious scientific achievements, Peter was a fun-loving, gregarious person, with a great sense of humor, who loved to explore and enjoy life to the fullest. One of his close colleagues (Alan Fried) can recount numerous times when laughter was nearly non-stop during a week-long diode laser conference held on a boat in 1993 on the Volga River in Russia. At that time Alan really got to know and truly appreciate Peter with his great sense of humor and his unique ability to laugh at circumstances that would have distressed many others. Peter described how his stateroom, which he shared with his lovely wife Regina, felt like a loud bar every time someone played the piano right next to their paper-thin stateroom wall. Peter and Alan shared numerous laughs at the small fish eyes in the fish-eye soup starring them in the face during mealtimes on the boat, as well as at the merchants pestering all of the scientists when the boat docked at various stops along the Volga in an effort to sell tourist goods. Instead of getting annoyed, Peter loved to toy with the merchants by asking endless questions about how their goods compared to others and why he should buy them. Peter was continuously looking for innovation, even where common people would not expect it. His last "odd" purchase was a self-cooling beer keg, which he acquired for his daughter Laura's 18th birthday celebration.

Enjoying life also meant that more often than not his wife Regina, and sometimes daughter Laura, would accompany Peter on his business travels. We vividly remember a very brave Laura Werle during the 2011 FLAIR-SIRIS (Stable Isotope Ratio Infrared Spectrometry) conference cruise on 
Lake Starnberg, barely 16 years old, translating the sightseeing descriptions of the ship's captain from German into English. Regina also accompanied Peter on his last conference tour and subsequent visit to Grenoble. His last working day before departing on their vacation was spent visiting the LAME laser laboratory. Peter clearly enjoyed the lively discussions and made a number of interesting observations and suggestions. Nothing indicated that disaster would strike that same evening.
The scientific community has truly lost a great visionary with Peter's passing. We can only hope that we as scientists can continue to live up to his high standards and innovativeness. Most importantly, we can all learn from Peter and his ability to laugh at difficult circumstances.

Peter, we cannot overstate how much we all miss you. 